\title{
ÉDITORIAL
}

\section{La capacité de l'humain à s'adapter}

Lorsque l'appel à contribution pour ce numéro fut lancé, le 3 mars 2020, sous le titre «L'ère de l'innov-action $\mathrm{RH}$ : la mise en valeur de l'humain ", personne ne s'attendait à ce que le monde soit sur le point de basculer quelques jours plus tard. Plus encore, une des premières phrases de cet appel était: " la nécessité de s'adapter aux contraintes et aux opportunités de son environnement ». À ce moment, les défis auxquels faisaient face les organisations se rapportaient plus à la rareté de main-d'œuvre et à d'autres défis qui nous semblent bien lointains en ce moment en comparaison avec ceux auxquels nous avons été confrontés.

Nous avons été en mesure de constater, durant la dernière année, à quel point les entreprises doivent s'ajuster pour survivre. La résilience dont a fait preuve la majorité des organisations démontre à quel point l'adaptation n'est pas qu'un concept vide de sens que l'on enseigne dans nos universités. La notion de télétravail, enseignée comme étant une possibilité à la disposition des entreprises, est devenue la réalité d'une majorité de travailleurs. Nous sommes devenus de friands utilisateurs de logiciels de réunions, logiciels que nous ignorions même l'existence quelques semaines auparavant.

Nous avons pu observer au cours des douze derniers mois la capacité d'innovation des entreprises. Celle-ci a fait figure d'instinct de survie pour plusieurs organisations, alors que pour d'autres, le choc a été trop grand et elles n'ont pas été en mesure d'y survivre. La recherche d'amélioration et d'innovation a été, tout au long de cette année 2020, l'obsession des entrepreneurs et des gestionnaires à travers la planète. L'être humain au travail a démontré sa grande capacité à composer avec un environnement pouvant changer très rapidement, voir même, un environnement hostile.

Bien que la dernière année en fut une exceptionnelle, l'importance de faire preuve d'innovation en matière de gestion sera toujours une préoccupation pour les entreprises voulant atteindre des niveaux de performance supérieurs. Durant les dernières années, les entreprises ont composé, entre autres, avec une rareté de maind'œuvre sans précédent, avec un taux de chômage à son plus bas niveau avoisinant le taux de plein emploi. De plus, la main-d'œuvre s'est également beaucoup diversifiée au cours des dernières années amenant de nouvelles réalités pour les gestionnaires qui ont dû faire preuve d'innovation et d'adaptation.

Dans ce numéro, plusieurs sujets seront abordés en lien avec le thème énoncé lors de l'appel à contribution. Les sujets variés touchent des préoccupations importantes en gestion des ressources humaines. Que ce soit la santé psychologique au travail ou les impacts de la COVID-19 sur les travailleurs, en passant par le télétravail, ces sujets demandent aux travailleurs, aux gestionnaires et aux entreprises une capacité d'adaptation et d'innovation permettant d'assurer une meilleure gestion du capital humain de nos organisations.

Il faut aussi profiter de l'occasion pour remercier les auteurs qui nous ont présenté des articles de qualité pour la réalisation de cette revue. Il ne faut pas non plus oublier les évaluateurs qui ont permis à ce numéro de voir le jour, ainsi que toutes les personnes impliquées, de près ou de loin, à la réalisation de ce quatrième numéro.

Bonne lecture!

\section{Guy Arcand, Université du Québec à Trois-Rivières Olivier Gagnon, Université du Québec à Chicoutimi}

Doi: 10.1522/radm.no4.1238 